\title{
Fitting the Itô Stochastic differential equation to the COVID-19 data in Turkey
}

\author{
Sevda Özdemir Çalıkuşu ${ }^{1 *}$, Fevzi Erdoğan² \\ ${ }^{1}$ Yuzuncu Y1l Unıversity, Özalp Vocational School, Turkey, sevdaozdemir@yyu.edu.tr, ORCID: 0000-0002-0238-2677 \\ ${ }^{2}$ Van Yuzuncu Yil University, Faculty of Economics and Administrative Sciences, Turkey, ORCID: 0000-0003-3745-0198
}

A B S T R A C T

In this study, COVID-19 data in Turkey is investigated by Stochastic Differential Equation Modeling (SDEM). Firstly, parameters of SDE which occur in mentioned epidemic problem are estimated by using the maximum likelihood procedure. Then, we have obtained reasonable Stochastic Differential Equation (SDE) based on the given COVID-19 data. Moreover, by applying Euler-Maruyama Approximation Method trajectories of SDE are achieved. The performances of trajectories are established by Chi-Square criteria. The results are acquired by using statistical software R-Studio.These results are also corroborated by graphical representation.

\section{ARTICLE INFO}

Research article

Received: 30.04 .2021

Accepted: 20.10.2021

Keywords:

Itô stochastic

differential equation,

Euler-Maruyama

approximation method,

Maximum likelihood

estimation method,

COVID-19 data

${ }^{*}$ Corresponding author

\section{Introduction}

Coronavirus disease (COVID-19) is an infectious disease caused by a newly discovered coronavirus. Most people infected with the COVID-19 virus will experience mild to moderate respiratory illness and recover without requiring special treatment. Older people, and those with underlying medical problems like cardiovascular disease, diabetes, chronic respiratory disease, and cancer are more likely to develop serious illness [1]. The first case of COVID-19 in Turkey was reported on 11 March 2020, and the number of reported cases has increased day by day.

Stochastic differential equations model (SDEM) stochastic evolution as time evolves. These models have a variety of applications in many disciplines and emerge naturally in the study of many phenomena. Examples of these applications are physics, astronomy, mechanics, economics, mathematical finance, geology, genetic analysis, ecology, cognitive psychology, neurology, biology, biomedical sciences, epidemiology, political analysis and social processes, and many other fields of science and engineering [2]. And it is known that SDEMs are more realistic mathematical model than normal differential equation models of the situation [3].
Because stochastic differential equations, unlike normal differential equations, contain random effects called "white noise".

In [4], Kostrista E. and Cibuku D. are looking for answers to some questions which are related with stochastic differential equations. These questions are 'In what situations does the SDE arise?', 'What are its essential features?', 'What are the applications and the connections to the other fields?' and 'How does SDEs model the physical situation and white noise process which is the generalized mean-square derivative of the Wiener process or Brownian motion?'. In [5], Ince N. and Shamilov A. develop a new method to obtain approximate probability density function (pdf) of random variable of solution of stochastic differential equations (SDEs) by using generalized entropy optimization methods (GEOM). By starting given statistical data and Euler-Maruyama (EM) method approximating SDE are constructed several trajectories of SDEs. The constructed trajectories allow to obtain random variable according to the fixed time. In [6], Mahrouf M. et al. extend the well-known SIR compartmental model to deterministic and stochastic time-delayed models in order to predict the epidemiological trend of COVID-19 in Morocco and to assess the potential role of multiple 
preventive measures and strategies imposed by Moroccan authorities. In [7], Bak J. Et al. propose a method to test for lack-of-fit of an estimated stochastic differential equation. The method is based on Monte Carlo simulation of trajectories between neighbour observations and, thus, it does not rely on the availability of explicit expressions of the conditional densities. Consequently, both non-linear models and models with state-dependent drift and diffusion can be handled. In [8], Rezaeyan R. and Farnoosh R. present an application of the continuous Kalman-Bucy filter for a RC circuit. The analytic solution of the resulting stochastic integral equations are found using the Ito formula. In [9], Ang examine the use of a simple stochastic differential equation in the modelling of an epidemic. Real data for the Singapore SARS outbreak are used for a detailed study. The model is solved numerically and implemented on matlab, with further analysis and refinement. This article is built around several matlab programs and serves to provide a practical and accessible introduction to numerical methods for a stochastic model for epidemics. In [10], Simha A. et al. model the region-wise trends of the evolution to COVID-19 infections using a stochastic SIR model. The SIR dynamics are expressed using Itô stochastic differential equations. In [11], Zhang et al. consider stochastic mathematical model for COVID-19. Firstly, the formulation of a stochastic susceptible-infected-recovered model is presented. Secondly, they devote with full strength our concentrated attention to sufficient conditions for extinction and persistence. Thirdly, they examine the threshold of the proposed stochastic COVID-19 model, when noise is small or large. Finally, they show the numerical simulations graphically using MATLAB.

Considering the studies mentioned above, the main features of the work finds an appropriate stochastic differential equation model for the given COVID-19 data. So in this research, we have examined by SDEM the COVID-19 data in Turkey between 11.03.2020 and 09.06.2020. In here, parameter values have been estimated from real data using the Maximum Likelihood procedure and approximate solutions of the established stochastic differential equation model have been obtained by using Euler-Maruyama method.

\section{Stochastic calculus}

\subsection{Probability Theory}

Stochastic differential equations provide a link between probability theory and the much older and more developed fields of ordinary and partial differential equations [4]. So, the terms of probability theory are significant in stochastic calculus. For instance, a stochastic integral is a random variable and the solution of a stochastic differential equation at any fixed time is a random variable [12]. Therefore, if anybody wants to learn about probability theory in detail, [3, 12-18] can be refered.

\subsection{Itô Stochastic Differential Equation}

Modeling physical systems by ordinary differential equations (ODEs) ignores stochastic effects. Addition of random elements into the differential equations leads to what is called stochastic differential equations (SDEs), and the term stochastic is called noise. A stochastic differential equation (SDE) is a differential equation in which one or more of the terms is a stochastic process, thus resulting in a solution which is itself a stochastic process [8].

A typical one dimensional Itô stochastic differential equation has the form

$$
\begin{aligned}
X(t, \omega)=X(0, \omega) & +\int_{0}^{t} f(s, X(s, \omega)) d s \\
& +\int_{0}^{t} g(s, X(s, \omega)) d W(s, \omega)
\end{aligned}
$$

and differential form

$$
d X(t)=f(t, X)+g(t, X) d W(t)
$$

for $0 \leq \mathrm{t} \leq \mathrm{T}$ with $\mathrm{X}(0, \cdot) \in \mathrm{H}_{\mathrm{RV}}, \mathrm{H}_{\mathrm{RV}}$ is the Hilbert space of random variables and $X(t, \omega)$ is a stochastic process not a deterministic function. $W(t, \omega)=W(t)$ is a Wiener process of Brownian motion and since it is nowhere differentiable. $\mathrm{W}(\mathrm{t}), \mathrm{t} \geq 0$ is a continuous stochastic process with stationary independent increments such that $W(0)=0$, $\int_{c}^{d} d W(s)=W(d)-W(c) \sim N(0, d-c)$ for all $0 \leq c \leq d$. The function $\mathrm{f}$ is often called the drift coefficient of the stochastic differential equation while $\mathrm{g}$ is referred to as the diffusion coefficient [12].

\section{Material and methods}

\subsection{Euler-Maruyama Method}

An Ito stochastic differential equation on the interval $[0, \mathrm{~T}]$ has the form

$$
d X(t)=f(t, X(t) ; \theta) d t+g(t, X(t) ; \theta) d W(t)
$$

is considered where $\theta \in \mathbb{R}^{\mathrm{m}}$ is a vector of parameters that are unknown for $0 \leq \mathrm{t} \leq \mathrm{T}$ where $\mathrm{X}(0)=\mathrm{X}_{0}, \mathrm{X}_{0} \in \mathrm{H}_{\mathrm{RV}}$ and $\mathrm{W}(\mathrm{t})$ is the Wiener process.

As the exact solution to a stochastic differential equation is generally difficult to obtain, it is useful to be able to approximate the solution. Euler-Maruyama method is a simple numerical method. When applied to (3.1), Euler's method has the form 


$$
\begin{gathered}
X_{j+1, i}^{(m)}\left(t_{i-1}+(j+1) \frac{\Delta t}{K}\right)=X_{j, i}^{(m)}\left(t_{i-1}+j \frac{\Delta t}{K}\right) \\
+f\left(t_{i-1}+j \frac{\Delta t}{K}, X_{j, i}^{(m)}\left(t_{i-1}+j \frac{\Delta t}{K}\right)\right) \frac{\Delta t}{K}+ \\
+g\left(t_{i-1}+j \frac{\Delta t}{K}, X_{j, i}^{(m)}\left(t_{i-1}+j \frac{\Delta t}{K}\right)\right) \sqrt{\frac{\Delta t}{K}} \eta_{j, i}^{(m)}
\end{gathered}
$$

for $\mathrm{i}=0,1,2, \ldots, \mathrm{N}-1$ and $\mathrm{j}=0,1,2, \ldots, \mathrm{K}-1$ where $\Delta \mathrm{t}=\frac{\mathrm{T}}{\mathrm{N}}, \mathrm{t}_{\mathrm{i}}=\mathrm{i} \Delta \mathrm{t}$, and $\Delta \mathrm{t}_{\mathrm{i}}=\mathrm{t}_{\mathrm{i}+1}-\mathrm{t}_{\mathrm{i}}=\frac{\Delta \mathrm{T}}{\mathrm{K}}, \mathrm{\eta}_{\mathrm{j}, \mathrm{i}}^{(\mathrm{m})} \sim \mathrm{N}(0, \Delta \mathrm{t})$, $\Delta \mathrm{W}(\mathrm{t}, \mathrm{w})=\mathrm{W}\left(\mathrm{t}_{\mathrm{i}+1}, \mathrm{w}\right)-\mathrm{W}\left(\mathrm{t}_{\mathrm{i}}, \mathrm{w}\right), \Delta \mathrm{W}(\mathrm{t}, \mathrm{w}) \sim \mathrm{N}\left(0, \Delta \mathrm{t}_{\mathrm{i}}\right)$, and $\mathrm{m}$ indicates a simulation number. This means that by changing number $\mathrm{K}, \mathrm{m}$ times then $\mathrm{m}$ simulations realized, [14].

In this research, the extreme values of approximation trajectories $\mathrm{X}_{\mathrm{j}+1, \mathrm{i}}^{(\mathrm{m})}$ by applying Euler-Maruyama Approximation Method are achieved.

\subsection{Parameter Estimation for Stochastic Differential Equations}

The problem is to find an estimate of the vector $\theta$ given these $\mathrm{N}+1$ data points. Two estimation methods are a maximum likelihood estimation method and a nonparametric estimation method, [12].

In this study, we use the maximum likelihood estimation method to estimate parameters from the real data.

\subsection{Maximum-Likelihood Estimation Method}

Let $\mathrm{p}\left(\mathrm{t}_{\mathrm{k}}, \mathrm{x}_{\mathrm{k}} \mid \mathrm{t}_{\mathrm{k}-1}, \mathrm{x}_{\mathrm{k}-1} ; \boldsymbol{\theta}\right)$ be the transition probability density of $\left(t_{k}, x_{k}\right)$ starting from $\left(t_{k-1}, x_{k-1}\right)$ given the vector $\boldsymbol{\theta}$. Suppose that the density of the initial state is $\mathrm{p}_{0}\left(\mathrm{x}_{0} \mid \boldsymbol{\theta}\right)$.

In maximum likelihood estimation of $\boldsymbol{\theta}$, the joint density

$$
\mathrm{D}(\boldsymbol{\theta})=\mathrm{p}_{0}\left(\mathrm{x}_{0} \mid \boldsymbol{\theta}\right) \prod_{\mathrm{k}=1}^{\mathrm{N}} \mathrm{p}\left(\mathrm{t}_{\mathrm{k}}, \mathrm{x}_{\mathrm{k}} \mid \mathrm{t}_{\mathrm{k}-1}, \mathrm{x}_{\mathrm{k}-1} ; \boldsymbol{\theta}\right)
$$

is maximized over $\boldsymbol{\theta} \in \mathbb{R}^{\mathbf{m}}$ [12]. However, to avoid small numbers on a computer, it is more convenient to minimize the function $\mathrm{L}(\boldsymbol{\theta})=-\ln (\mathrm{D}(\boldsymbol{\theta}))$ which has the form

$$
\mathrm{L}(\boldsymbol{\theta})=-\ln \left(\mathrm{p}_{0}\left(\mathrm{x}_{0} \mid \boldsymbol{\theta}\right)\right)-\sum_{\mathrm{k}=1}^{\mathrm{N}} \ln \left(\mathrm{p}\left(\mathrm{t}_{\mathrm{k}}, \mathrm{x}_{\mathrm{k}} \mid \mathrm{t}_{\mathrm{k}-1}, \mathrm{x}_{\mathrm{k}-1} ; \boldsymbol{\theta}\right)\right) .
$$

\section{A Stochastic Model for a COVID-19 outbreak}

A simple stochastic model for a disease such as COVID-19 that spreads very quickly is given by the stochastic differential equation

$$
\begin{gathered}
d X(t)=\theta_{1} X(t)(n-X(t)) d t+\theta_{2} X(t) d W(t), \\
X(0)=X_{0}, \quad 0 \leq t \leq T
\end{gathered}
$$

where $\mathrm{X}(\mathrm{t})$ is the number of infected and susceptible individuals at time $t$ (days), $\theta_{1}$ and $\theta_{2}$ are real constants, $n$ is the total number of individuals in that community and $W(t)$ is a random variable representing a standard Wiener process. In here, it is obvious that drift represents the deterministic portion of the model, while diffusion represents the stochastic component [9].

In this study, we use equation (3.3) in the modeling of an infectious disease, using the outbreak of COVID-19 data in Turkey between 11.03.2020 and 09.06.2020.

\section{An application}

One of the important application areas of stochastic differential equations is population biology such as epidemic model. In this study, the COVID-19 data in Turkey between 11.03.2020 and 09.06.2020 are examined. This data set is accessed with the help of World Health Organization (WHO) shared the link via https://www.who.int/ [1], and the data set is shown in Table 1. 
Table 1. The COVID-19 data in Turkey between 11.03.2020 and 09.06.2020

\begin{tabular}{|c|c|c|c|c|c|c|c|}
\hline Day & $\begin{array}{c}\text { Total number } \\
\text { of confirmed } \\
\text { cases }\end{array}$ & Day & $\begin{array}{c}\text { Total number } \\
\text { of confirmed } \\
\text { cases }\end{array}$ & Day & $\begin{array}{c}\text { Total number } \\
\text { of confirmed } \\
\text { cases }\end{array}$ & Day & $\begin{array}{c}\text { Total number } \\
\text { of confirmed } \\
\text { cases } \\
\end{array}$ \\
\hline 1 & 1 & 24 & 20921 & 47 & 110130 & 70 & 151615 \\
\hline 2 & 1 & 25 & 23934 & 48 & 112261 & 71 & 152587 \\
\hline 3 & 5 & 26 & 27069 & 49 & 114653 & 72 & 153548 \\
\hline 4 & 5 & 27 & 30217 & 50 & 117589 & 73 & 154500 \\
\hline 5 & 5 & 28 & 34109 & 51 & 120204 & 74 & 155686 \\
\hline 6 & 47 & 29 & 38226 & 52 & 122392 & 75 & 156827 \\
\hline 7 & 47 & 30 & 42282 & 53 & 124375 & 76 & 157814 \\
\hline 8 & 191 & 31 & 47029 & 54 & 126045 & 77 & 158762 \\
\hline 9 & 191 & 32 & 52167 & 55 & 127659 & 78 & 159797 \\
\hline 10 & 670 & 33 & 56956 & 56 & 129491 & 79 & 160979 \\
\hline 11 & 947 & 34 & 61049 & 57 & 131744 & 80 & 162120 \\
\hline 12 & 1236 & 35 & 65111 & 58 & 133721 & 81 & 163103 \\
\hline 13 & 1529 & 36 & 69392 & 59 & 135569 & 82 & 163942 \\
\hline 14 & 1872 & 37 & 74193 & 60 & 137115 & 83 & 164769 \\
\hline 15 & 2433 & 38 & 78546 & 61 & 138657 & 84 & 165555 \\
\hline 16 & 3629 & 39 & 82329 & 62 & 139771 & 85 & 166422 \\
\hline 17 & 5698 & 40 & 86306 & 63 & 141475 & 86 & 167410 \\
\hline 18 & 7402 & 41 & 90980 & 64 & 143114 & 87 & 168340 \\
\hline 19 & 9271 & 42 & 95591 & 65 & 144749 & 88 & 169218 \\
\hline 20 & 10827 & 43 & 98674 & 66 & 146457 & 89 & 170132 \\
\hline 21 & 13531 & 44 & 101790 & 67 & 148067 & 90 & 171121 \\
\hline 22 & 15679 & 45 & 104912 & 68 & 149435 & 91 & 172114 \\
\hline 23 & 18135 & 46 & 107773 & 69 & 150593 & & \\
\hline
\end{tabular}

We shall use following stages of investigation to solve our problem. Firstly, the parameters of SDE established for the COVID-19 data that mentioned above are estimated by using maximum likelihood estimation method. Then, we have obtained reasonable Stochastic Differential Equation (SDE) based on the given COVID-19 data. Moreover, by applying Euler-Maruyama Approximation Method trajectories of SDE are achieved and they are demonstrated in Figure 1.

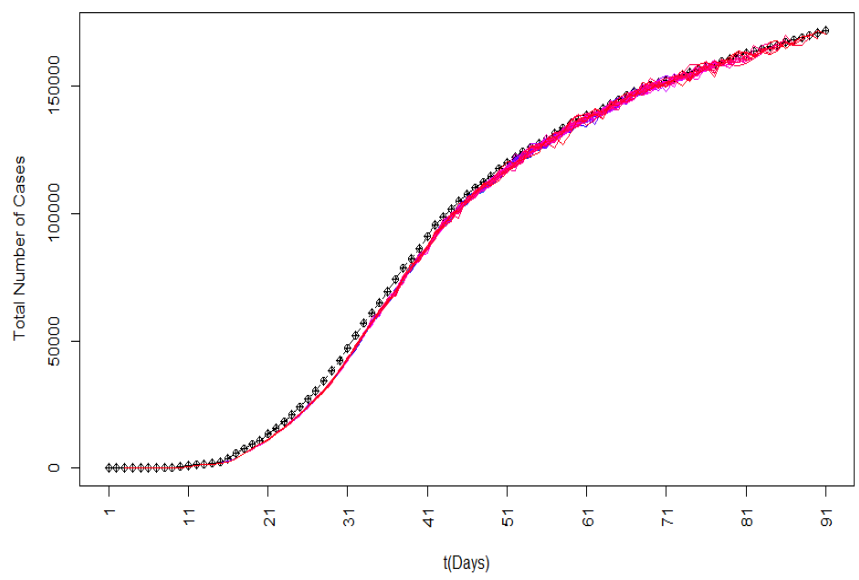

Figure 1. The COVID-19 data in Turkey between 11.03.2020 and 09.06.2020 (black line) and approximate EM trajectories (colored lines) of the SDE
Using the maximum likelihood estimation method, $\hat{\theta}_{1}=$ 0.000004 and $\hat{\theta}_{2}=0.98$ are obtained. If these estimated parameters are considered in the equation (3.3), $\mathrm{dX}(\mathrm{t})$ has the following form

$$
\begin{array}{r}
d X(t)=0.000004 X(t)(172114-X(t)) d t \\
+0.98 X(t) d W(t), \quad X(0)=1
\end{array}
$$

Hence, a reasonable stochastic differential equation based on COVID-19 data is achieved. Furthermore, the approximate EM values of the data set $\widehat{X}\left(t_{i}\right), i=1,2, \ldots, 91$ are obtained corresponding to the COVID-19 data in Turkey between 11.03.2020 and 09.06.2020. Approximate trajectories taken randomly according to $\widehat{X}\left(t_{i}\right)$ for $i=91$ is given in Table 2 .

The performances of SDE are established by Chi-Square criteria. The results are acquired by using statistical software R-Studio. These results are also corroborated by graphical representation in Figure 2. 
Table 2. The COVID-19 data in Turkey between 11.03.2020 and 09.06.2020 and the approximate $\hat{X}\left(t_{91}\right)$ EM values of the data set

\begin{tabular}{|c|c|c|c|c|c|c|c|}
\hline $\begin{array}{c}\text { Total } \\
\text { number of } \\
\text { confirmed } \\
\text { cases }\end{array}$ & EM & $\begin{array}{c}\text { Total } \\
\text { number of } \\
\text { confirmed } \\
\text { cases }\end{array}$ & EM & $\begin{array}{c}\text { Total } \\
\text { number of } \\
\text { confirmed } \\
\text { cases }\end{array}$ & EM & $\begin{array}{c}\text { Total } \\
\text { number of } \\
\text { confirmed } \\
\text { cases }\end{array}$ & EM \\
\hline 1 & 1.0 & 20921 & 20979.3 & 110130 & 110413.4 & 151615 & 150807.5 \\
\hline 1 & 1.0 & 23934 & 24225.2 & 112261 & 113325.3 & 152587 & 152389.3 \\
\hline 5 & 5.1 & 27069 & 27249.7 & 114653 & 113652.6 & 153548 & 153784.1 \\
\hline 5 & 5.0 & 30217 & 29969.5 & 117589 & 117343.4 & 154500 & 155002.8 \\
\hline 5 & 5.0 & 34109 & 34189.8 & 120204 & 121549.8 & 155686 & 156362.2 \\
\hline 47 & 46.8 & 38226 & 38414.4 & 122392 & 124744.7 & 156827 & 154897.3 \\
\hline 47 & 47.3 & 42282 & 42731.3 & 124375 & 125322.3 & 157814 & 158103.2 \\
\hline 191 & 193.6 & 47029 & 47344.9 & 126045 & 127087.2 & 158762 & 162560.7 \\
\hline 191 & 193.0 & 52167 & 52638.2 & 127659 & 127618.1 & 159797 & 160550.4 \\
\hline 670 & 684.4 & 56956 & 56883.4 & 129491 & 131721.1 & 160979 & 164169.5 \\
\hline 947 & 944.5 & 61049 & 61073.1 & 131744 & 131106.9 & 162120 & 162024.3 \\
\hline 1236 & 1266.3 & 65111 & 66423.3 & 133721 & 134270.7 & 163103 & 163335.7 \\
\hline 1529 & 1526.7 & 69392 & 70377.5 & 135569 & 135853.1 & 163942 & 164039.0 \\
\hline 1872 & 1893.8 & 74193 & 74002.2 & 137115 & 133322.9 & 164769 & 163312.3 \\
\hline 2433 & 2451.1 & 78546 & 80126.2 & 138657 & 137647.4 & 165555 & 162744.7 \\
\hline 3629 & 3730.0 & 82329 & 84909.8 & 139771 & 138586.6 & 166422 & 167981.2 \\
\hline 5698 & 5693.9 & 86306 & 87982.4 & 141475 & 139412.4 & 167410 & 166266.3 \\
\hline 7402 & 7416.3 & 90980 & 91190.2 & 143114 & 144217.7 & 168340 & 166437.7 \\
\hline 9271 & 92478.0 & 95591 & 95935.5 & 144749 & 147099.5 & 169218 & 169196.8 \\
\hline 10827 & 10909.2 & 98674 & 100407.8 & 146457 & 147548.3 & 170132 & 171263.2 \\
\hline 13531 & 13660.9 & 101790 & 101573.0 & 148067 & 146751.3 & 171121 & 170532.4 \\
\hline 15679 & 15759.2 & 104912 & 106494.3 & 149435 & 149706.2 & 172114 & 172661.0 \\
\hline 18135 & 18290.2 & 107773 & 107175.1 & 150593 & 149324.4 & & \\
\hline
\end{tabular}

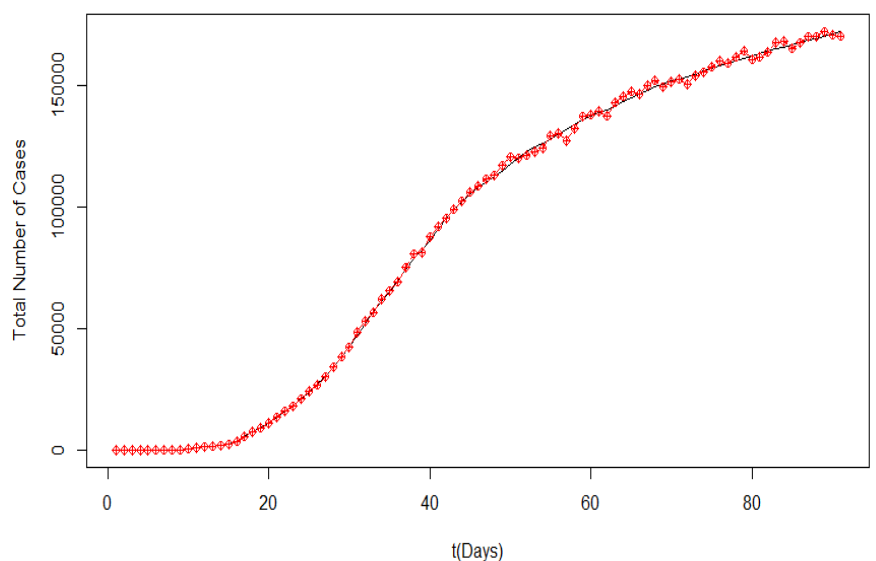

Figure 2. The COVID-19 data in Turkey between 11.03.2020 and 09.06.2020 (black line) and its EM trajectory starting from $\hat{X}\left(t_{91}\right)$ (red line)

\section{Conclusion}

In this study COVID-19 data in Turkey between 11.03.2020 and 09.06.2020 is investigated by Stochastic Differential Equation Modeling (SDEM). Firstly, parameters of SDE established for the COVID-19 data that mentioned above are estimated estimated $\hat{\theta}_{1}=0.000004$ and $\hat{\theta}_{2}=0.98$ by using maximum likelihood estimation method. Then, we have obtained reasonable Stochastic Differential Equation (SDE) based on the given data. Moreover, by applying EulerMaruyama Approximation Method trajectories of SDE are achieved. The acceptancy of equation (3.3) with parameters $\theta_{1}$ and $\theta_{2}$ to consider in our study for COVID-19 data in Turkey is provided with Chi-square value equal to 7735 of Goodness of fit test. The performances of trajectories according to $\widehat{\mathrm{X}}\left(\mathrm{t}_{91}\right)$ is established by Chi-Square criteria. The acceptancy of Chi-Square criteria for $\widehat{X}\left(t_{91}\right)$ is realized by values of $p_{\text {value }}=0.2451$ and we do not reject the null hypothesis that given COVID-19 data is fit to last values of EM estimations. As a result, the SDEM we established fits well with COVID-19 data Turkey between 11.03.2020 and 09.06.2020. 


\section{References}

[1]. World Health Organization (WHO). Coronavirus. Available from: https://www.who.int/healthtopics/coronavirus\#tab=tab_1 (Accessed: April 15, 2021).

[2]. Iacus S.M., Simulation and Inference for Stochastic Differential Equations with R Examples. USA: Springer, 2008.

[3]. Oksendal B., Stochastic Differential Equations an Introduction with Applications, 5th ed., Corrected Printing. New York: Springer-Verlag Heidelberg, 2003.

[4]. Kostrista E., Çibuku D., "Introduction to Stochastic Differential Equations", Journal of Natural Sciences and Mathematics of UT, 3, (2018), 5-6.

[5]. Ince N., Shamilov A., "An application of new method to obtain probability density function of solution of stochastic differential equations", Applied Mathematics and Nonlinear Sciences, 5.1 (2020), 337-348.

[6]. Mahrouf M. et al. "Modeling and forecasting of COVID-19 spreading by delayed stochastic differential equations", Axioms 10.1, (2021), 18.

[7]. Bak J., Nielsen A. and Madsen H., "Goodness of fit of stochastic differential equations", 21 th Symposium I Anvendt Statistik, Copenhagen Business School, Copenhagen, Denmark. 1999.

[8]. Rezaeyan R., Farnoosh R., "Stochastic Differential Equations and Application of the Kalman-Bucy Filter in the Modeling of RC Circuit", Applied Mathematical Sciences, 4, (2010), 1119-1127.
[9]. Ang K. C., "A Simple Stochastic Model for an Epidemic-Numerical Experiments with MATLAB", The Electronic Journal of Mathematics and Technology, 1, (2007), 117-128.

[10]. Simha A., Prasad R.V., and Narayana A.,. "A simple stochastic sir model for covid 19 infection dynamics for karnataka: Learning from europe." arXiv preprint arXiv:2003.11920, (2020).

[11]. Zhang Z., et al. "Dynamics of COVID-19 mathematical model with stochastic perturbation." Advances in Difference Equations, 2020.1, (2020), 1-12.

[12]. Allen E., Modeling with Itô Stochastic Differential Equations. USA: Springer, 2007.

[13]. Shamilov A., Measurement Theory, Probability and Lebesgue Integral, Eskişehir: Anadolu University Publications, 2007.

[14]. Shamilov A., Differential Equations with Theory and Solved Problems, Turkey: Nobel Publishing House, 2012.

[15]. Shamilov A., Probability Theory with Conceptional Interpretations and Applications. Turkey: Nobel Publishing House, 2014.

[16]. Andersson H., Britton T., Stochastic Epidemic Models and Their Statistical Analysis. New York: Springer, 2000 .

[17]. Allen L.J.S., An Introduction to Stochastic Processes with Applications to Biology. Upper Saddle River, New Jersey: Pearson Education Inc., 2003.

[18]. Higham D.J., “An Algorithmic Introduction to Numerical Simulation of Stochastic Differential Equations”, SIAM Review, 43, (2001), 525-546. 